\title{
Comparison of a specific HPLC determination of toxic aconite alkaloids in processed Radix aconiti with a titration method of total alkaloids
}

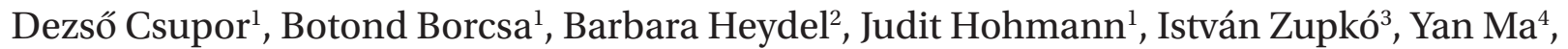 \\ Ute Widowitz ${ }^{2}$, and Rudolf Bauer ${ }^{2}$ \\ ${ }^{1}$ Department of Pharmacognosy, University of Szeged, Szeged, Hungary, ${ }^{2}$ Institute of Pharmaceutical Sciences, \\ Department of Pharmacognosy, Karl-Franzens University, Graz, Austria, ${ }^{3}$ Department of Pharmacodynamics and \\ Biopharmacy, University of Szeged, Szeged, Hungary, and ${ }^{4}$ Department of Pathophysiology, Center of Physiology, \\ Pathophysiology and Immunology, Medical University, Vienna, Austria
}

\begin{abstract}
Context: In traditional Chinese medicine, Aconitum (Ranunculaceae) roots are only applied after processing. Nevertheless, several cases of poisoning by improperly processed aconite roots have been reported.

Objective: The aim of this study was to develop a reliable analytical method to assess the amount of toxic aconite alkaloids in commercial aconite roots, and to compare this method with the commonly used total alkaloid content determination by titration.

Materials and methods: The content of mesaconitine, aconitine, and hypaconitine in 16 commercial samples of processed aconite roots was determined by an HPLC method and the total alkaloid content by indirect titration. Five samples were selected for in vivo toxicological investigation.

Results: In most of the commercial samples, toxic alkaloids were not detectable, or only traces were found. In four samples, we could detect $>0.04 \%$ toxic aconite alkaloids, the highest with a content of $0.16 \%$. The results of HPLC analysis were compared with the results obtained by titration, and no correlation was found between the two methods. The in vivo results reassured the validity of the HPLC determination.

Discussion and conclusion: Samples with mesaconitine, aconitine, and hypaconitine content below the HPLC detection limit still contained up to $0.2 \%$ alkaloids determined by titration. Since titration of alkaloids gives no information selectively on the aconitine-type alkaloid content and toxicity of aconite roots this method is not appropriate for safety assessment. The HPLC method developed by us provides a quick and reliable assessment of toxicity and should be considered as a purity test in pharmacopoeia monographs.
\end{abstract}

Keywords: Processed aconite root, toxic aconite alkaloids, HPLC determination, titration method, Aconitum carmichaeli, Aconitum kusnezoffii

\section{Introduction}

Aconitum carmichaeli Debx. and Aconitum kusnezoffii Reichb. (Ranunculaceae) have been widely used in the system of traditional Chinese medicine (TCM), primarily for analgesic purposes. Radix aconiti (dried mother root tuber of A. carmichaeli Debx., Chinese name: Chuanwu) and its processed form (Zhichuanwu), Radix aconiti lateralis praeparata (processed daughter root of A. carmichaeli Debx., Chinese name: Fuzi), Radix aconiti kusnezoffii (dried root tuber of A. kusnezoffii Reichb., Chinese name: Caowu), and its processed form (Zhicaowu), and Folium aconiti kusnezoffii (Caowuye) are listed in the Pharmacopoeia of the People's Republic of China (Chinese Pharmacopoeia Commission, 2005). Unprocessed aconite roots are very toxic due to the presence of diterpene-diester alkaloids (Xiao et al., 2006);

Address for Correspondence: Dezső Csupor, Department of Pharmacognosy, University of Szeged, Eötvös u. 6., Szeged, H-6720 Szeged, Hungary. Tel: +36-62-546451. E-mail: csupor.dezso@pharmacognosy.hu

(Received 10 May 2011; revised 0000 0000; accepted 05 June 2011) 
therefore, Radix aconiti and Radix aconiti kusnezoffii are allowed to be applied only after processing.

The main toxic compounds of $A$. kusnezoffii and $A$. carmichaelii are the aconitine-type diterpene alkaloids aconitine, mesaconitine, and hypaconitine (Figure 1). These compounds possess high affinity to the site II of $\mathrm{Na}^{+}$channels, the ability to enhance $\left[\mathrm{Na}^{+}\right]_{\mathrm{i}}$ and $\left[\mathrm{Ca}^{2+}\right]_{i}$, a strong arrhythmogenic action that starts at about $30 \mathrm{nM}$, and high acute toxicity $\left(\mathrm{LD}_{50}\right.$ values about $0.15 \mathrm{mg} /$ $\mathrm{kg}$ ) (Gutser et al., 1998). The main causes of death are refractory ventricular arrhythmias and asystole and the overall in-hospital mortality is $5.5 \%$. In Asia, aconite poisoning is much more common because of the continued use of aconite roots in traditional medicine. However, with easy access to Asian herbal medicines in western societies, aconite poisoning can occur anywhere in the world (Chan, 2009).

Processing reduces toxicity because of hydrolysis of the ester groups of aconitine-type alkaloids (Chan, 2009). First, the acetyl group is hydrolyzed and in the second step the benzoyl group is hydrolyzed (Figure 2). Since the benzoyl ester is more stable, in the properly processed plant material the concentration of benzoylaconine, benzoylmesaconine, and benzoylhypaconine is remarkable. The $\mathrm{LD}_{50}$ values of aconitine in mice are $1.80 \mathrm{mg} / \mathrm{kg}$ p.o., $0.31 \mathrm{mg} / \mathrm{kg}$ i.p., and $0.12 \mathrm{mg} / \mathrm{kg}$ i.v., of benzoylaconine $1500 \mathrm{mg} / \mathrm{kg}$ p.o., $70 \mathrm{mg} / \mathrm{kg}$ i.p., and $23 \mathrm{mg} / \mathrm{kg}$ i.v., and of aconine $120 \mathrm{mg} / \mathrm{kg}$ i.v. (Wada et al., 2006). Aconitum roots also contain the so-called lipoalkaloids, which are compounds esterified with fatty acids in the C-8 position. Lipo-alkaloids are more stable than aconitine-type alkaloids and can be detected both in unprocessed and in processed roots (Wang et al., 2002, 2003). Lipo-alkaloids are substantially less toxic than aconitine-type compounds as evidenced by the difference in the lethal intravenous single doses ( $>10 \mathrm{mg} / \mathrm{kg}$ for lipo-alkaloids) (Csupor et al., 2009). Due to insufficient control, several cases of poisoning have been reported (Singhuber et al., 2009), that may be the result of diverse alkaloid content of the starting material, unproper processing, and not least the lack of appropriate quality control.

Quality control criteria of pharmacopoeias are not always sufficient to warrant safety. In case of Radix aconiti and Radix aconiti kusnezoffii, the diterpene alkaloid content is not limited in the Chinese Pharmacopoeia and the warning of the Chinese Pharmacopoeia ("be cautious about the unprocessed root taken orally") is not commensurable to the danger of toxicity. In the Radix aconiti praeparata and Radix aconiti kusnezoffii praeparata monographs, a rather complicated colorimetric assay is used for the determination of diester alkaloids (required content level should not be $>0.15 \%$ ), and a titrimetric assay for the determination of the total alkaloid content (required level should not be $<0.20 \%$ of alkaloids, calculated as aconitine). The dosage of these two herbal drugs is $1.5-3 \mathrm{~g}$, which may contain as much as $4.5 \mathrm{mg}$ diestertype alkaloids (Chinese Pharmacopoeia Commission, 2005). Moreover, for Radix aconiti lateralis praeparata, the dose of which is $3-15 \mathrm{~g}$, only a quantitatively not too reliable TLC analysis of aconitine is specified (Chinese Pharmacopoeia Commission, 2005). Taking into account that the minimum lethal dose of aconitine is $3-6 \mathrm{mg}$ (Caravati et al., 2004), it is obvious that only careful
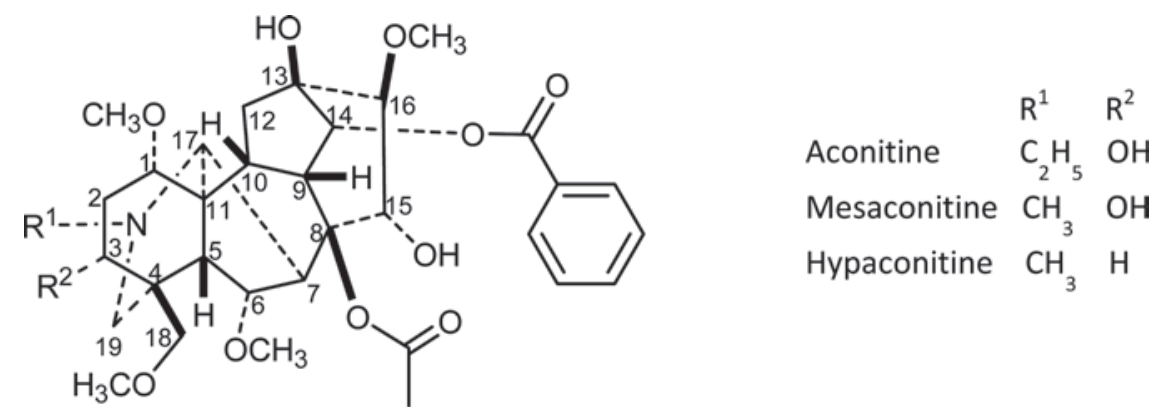

Figure 1. The main toxic Aconitum diterpene alkaloids.

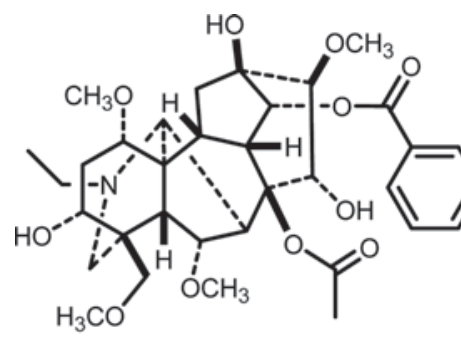

Aconitine

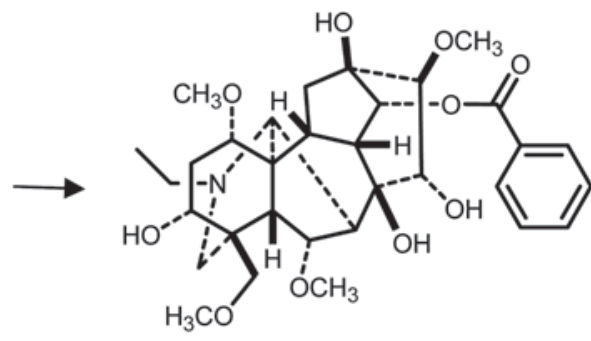

14-Benzoylaconine

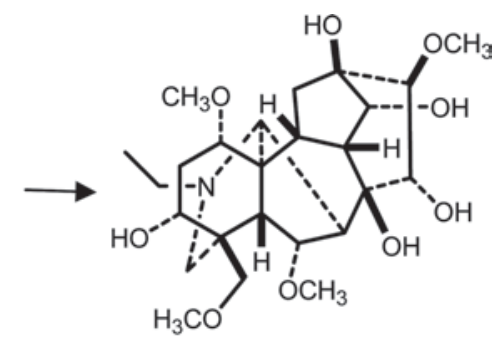

Aconine

Figure 2. Decomposition of aconitine during processing of Aconitum drugs. 
processing and quality control may warrant the safety of aconite containing medicinal products.

There are no Aconitum monographs neither in the U.S. Pharmacopoeia (USP) nor in the European Pharmacopoeia (Ph. Eur.). However, Aconitum napellus is included in the Swiss Pharmacopoeia (Pharmacopoeia Helvetica, 1988) and the monographs for Aconiti carmichaeli radix, Aconiti radix praeparata, Aconiti radix lateralis praeparata, Aconiti kusnezoffii radix praeparata, and Aconitum napellus and praeparationes homeopathicas are under preparation according to the on-line database of Ph. Eur (EDQM homepage). Since A. napellus has been applied in homeopathy, several homeopathic pharmacopoeias contain its monograph. However, the essence of these methods as demonstrated in the most prominent homeopathic pharmacopoeia, the German Homeopathic Pharmacopoeia (2005), is the titrimetric determination of total alkaloid content (German Homeopathic Pharmacopoeia, 2005).

Since the titrimetric determination provides no direct information on the toxic diester-type alkaloid content of aconite roots, more reliable methods are required for the quality control of processed plant material. The goal of our work was to develop a quick and simple HPLC method for the quality control of aconite roots with comparable or better reliability to those of the previously published methods (Jiang et al., 2005; Xie et al., 2005; Wang et al., 2006; Kang et al., 2010), to compare the results of titration method with HPLC analysis of the toxic alkaloids, and to assess the reliability of these methods using an in vivo toxicity test.

\section{Methods}

The content of mesaconitine, aconitine, and hypaconitine has been determined in 16 commercial samples of processed aconite roots by HPLC and alkaloid titration.

Processed Aconitum samples were obtained from different suppliers (see Table 1).Unprocessed A. carmichaelii roots (one sample) were obtained from a pharmacy in China. Aconitine, mesaconitine, and hypaconitine were purchased from PhytoLab GmbH, Vestenbergsgreuth, Germany.

Acetonitrile (Merck) was of HPLC grade. All other chemicals were purchased from Spektrum-3D Ltd., Hungary. HPLC analysis was carried on a Waters HPLCDAD system equipped with Waters 2998 Photodiode Array Detector.

For HPLC, an improved version of a recently published method (Csupor et al., 2009) has been applied. In brief, $1 \mathrm{~g}$ roughly powdered drug was ground, passed through a $0.315 \mathrm{~mm}$ sieve, extracted with $5 \mathrm{~mL}$ of $1 \% \mathrm{HCl}$ solution for $20 \mathrm{~min}$ at room temperature in an ultrasonic bath. After centrifugation ( $10 \mathrm{~min}$ at $4500 \mathrm{rpm})$, the filtered $(0.45 \mu \mathrm{m}$ filter membrane) supernatant was analyzed by HPLC (column: Phenomenex Gemini $5 \mu \mathrm{m}, 0.1 \mathrm{~m} \times 4.6 \mathrm{~mm}$; mobile phase: A: $10 \mathrm{mM}$ aqueous $\mathrm{NH}_{4} \mathrm{HCO}_{3}$ buffer $\mathrm{pH} 10 \pm 0.2$ with $28 \%$ ammonia solution, B: acetonitrile; 0 min: $70 \%$
Table 1. Origin of investigated aconite roots.

\begin{tabular}{|c|c|}
\hline Code & Simplified code for HPLC analyses \\
\hline \multicolumn{2}{|c|}{ Zhicaowu-Aconiti kusnezoffi praeparata } \\
\hline 37951 & B3 (EDQM) \\
\hline 37747 & B4 (EDQM) \\
\hline- & B6 (Hong Kong Baptist University) \\
\hline \multicolumn{2}{|c|}{ Zhichuanwu (Shanxi)_Aconiti praeparata (radix) } \\
\hline 32969 & D1 (EDQM) \\
\hline- & D2 (Hong Kong Baptist University) \\
\hline \multicolumn{2}{|c|}{ Aconiti carmichaeli radix praeparata/Aconiti radix praeparata } \\
\hline 28479 & E1 (EDQM) \\
\hline 36890 & E2 (EDQM) \\
\hline \multicolumn{2}{|c|}{$\begin{array}{l}\text { Shanghai market-Aconiti radix praeparata/Aconiti radix } \\
\text { lateralis praeparata }\end{array}$} \\
\hline 37271 & $\mathrm{~F} 2-2003$ \\
\hline 37270 & F3-2004 \\
\hline 37269 & $\mathrm{~F} 4-2005$ \\
\hline 37275 & F5-2008 \\
\hline 37272 & F6-2009 \\
\hline \multicolumn{2}{|c|}{$\begin{array}{l}\text { German market-Aconiti radix praeparata/Aconiti radix lateralis } \\
\text { praeparata }\end{array}$} \\
\hline 37276 & G1-2004 \\
\hline 37265 & G2-2004 \\
\hline 37263 & G4-2007 \\
\hline 37266 & G5-2007 \\
\hline
\end{tabular}

A, $4 \min : 60 \%$ A, $20 \min : 55 \%$ A, $30 \min : 20 \%$ A, $35 \mathrm{~min}:$ $70 \% \mathrm{~A}$; flow rate: $1.1 \mathrm{~mL} / \mathrm{min}$, detection: $233 \mathrm{~nm}$, injected sample volume: $20 \mu \mathrm{L}$ ). Peaks of mesaconitine, aconitine, and hypaconitine were identified by comparison of the HPLC-DAD chromatograms of the extracts of aconite roots with the HPLC-DAD chromatograms of reference solutions. Alkaloid content was calculated by comparison of the sum of the areas under curves (AUC) of mesaconitine, aconitine, and hypaconitine on the basis of the calibration curve established for aconitine. Calibration was established for aconitine based on five concentrations (with a range of $0.05-1.625 \mu \mathrm{g}$ ).

Alkaloid titration was carried out according the method of the German Homeopathic Pharmacopoeia (2005). To $2 \mathrm{~g}$ of the powdered drug, $8 \mathrm{~mL}$ of $1 \% \mathrm{HCl}$ solution was added and extracted for $20 \mathrm{~min}$ at room temperature in an ultrasonic bath. After centrifugation, the supernatant was filtered and washed with $1 \mathrm{~mL}$ of $1 \% \mathrm{HCl}$ solution. Diluted ammonia was added to the filtrate until the mixture became alkaline. $\mathrm{CHCl}_{3}(60 \mathrm{~g})$ was added to the extract and shaken for $15 \mathrm{~min}$, then $2 \mathrm{~g}$ of freshly powdered tragacanth was added, shaken again, and filtered into a dry flask. The filtrate was concentrated to a few milliliters; $10 \mathrm{~mL}$ of $0.01 \mathrm{M} \mathrm{HCl}$ and $5 \mathrm{~mL}$ of $\mathrm{H}_{2} \mathrm{O}$ were added; and the remainder of the $\mathrm{CHCl}_{3}$ was evaporated. This solution was titrated with $0.01 \mathrm{M} \mathrm{NaOH}$ using methyl red as indicator.

To assess the relation of alkaloid content and toxicity, after the quantitative analysis five samples were selected for in vivo toxicological investigation. The extracts of these drugs were dissolved in physiological saline containing 5\% dimethyl sulfoxide and intraperitoneally 
administered to male CFLP mice (24-30g). Two of the tested extracts (E2, F5) were administered at three doses (equivalent to 240,800 , and $2400 \mathrm{mg}$ of drug/ $\mathrm{kg}$ ), whereas the others (B3, D, G2) were given only at the highest dose for 5-5 animals. Mice were observed $5 \mathrm{~min}, 2 \mathrm{~h}$, and $6 \mathrm{~h}$ after administration as well as once a day for five additional days. The experimental protocol satisfied the Guidelines for Animal Experimentation approved by the Animal Experimentation Committee of the University of Szeged.

\section{Results and discussion}

The developed HPLC method provides good separation of the main toxic alkaloids (Figure 3 ) and therefore serves as basis for reliable quantitative analysis. For quantitative determination, linear regression analysisfor aconitinewas performed by the external standard method. The regression equation for aconitine was $y=2999256695.1674 x+$ 66930.4245 ( $x$ stands for the amount of injected alkaloid in $\mathrm{mg}, y$ denotes the area under the curve). The correlation coefficient $\left(R^{2}\right)$ was 0.9994 . Using the HPLC method, in most of the samples no toxic alkaloids or only traces could be detected (Table 2). However, in four samples (D1, E2, F5, G4), $>0.04 \%$ of aconitine, hypaconitine, and mesaconitine, the highest with a content of $0.162 \%$ (E2), were quantified. The alkaloid content of these samples is high enough to question their safety.

The alkaloid content determined by titration was considerably higher than determined by HPLC. Samples, in which toxic alkaloids were not detectable by HPLC, still contained up to $0.2 \%$ alkaloids according to titration. Careful processing obviously degrades the toxic alkaloids but does not remove all alkaloids. Total alkaloid titration determines not only the toxic diester alkaloids but also the monoester, unesterified, and lipo-alkaloids. There was no correlation between the low values $(<0.02 \%$ HPLC), but there was a correlation in high values of alkaloid contents determined by the two methods (Figure 4). When taking into consideration only samples with measurable alkaloid content by HPLC, a correlation coefficient of 0.91 was found between the results of the two methods.

Acute toxicity of batches (E2, F5) high in diterpene alkaloids has been confirmed in CFLP mice. Extract of E2 proved to be the most toxic batch resulting in death of two out of five mice at the highest dose. These fatal cases were preceded by intensive skeletal muscle cramps and shortness of breath. Similar excitatory skeletal symptoms were characteristic for all groups except for G2. An intensive defecation was observed on mice treated with extracts E2 and F5. The next day and later on, all surviving animals were in good condition exhibiting no obvious toxic symptoms.

Although the pharmacological characteristics of Aconitum diterpene alkaloids have been extensively studied (Ameri, 1998), the exact mode of action of traditionally applied aconite drugs has not been elucidated so

Table 2. Content of alkaloids determined by titration and by HPLC analysis of toxic alkaloids (sum of mesaconitine, aconitine, and hypaconitine).

\begin{tabular}{llc}
\hline Code & HPLC (\%) & Titration (\%) \\
\hline \multicolumn{2}{l}{ Radix aconiti kusnezoffii praeparata } & \\
B3 & Not detectable & 0.065 \\
B4 & 0.007 & 0.013 \\
B6 & Not detectable & 0.194
\end{tabular}

Radix aconiti carmichaeli praeparata/Radix aconiti lateralis praeparata

$\begin{array}{lll}\mathrm{D} 1 & 0.054 & 0.207\end{array}$

D2 Not detectable $\quad 0.120$

$\begin{array}{lll}\text { E1 } & 0.013 & 0.062\end{array}$

E2 $0.162 \quad 0.433$

F2 Not detectable $\quad 0.045$

F3 Not detectable $\quad 0.103$

F4 Not detectable $\quad 0.123$

F5 $0.125 \quad 0.342$

F6 Not detectable $\quad 0.070$

G1 $0.003 \quad 0.113$

G2 $0.026 \quad 0.129$

$\begin{array}{lll}\mathrm{G} 4 & 0.043 & 0.097\end{array}$

G5 $0.011 \quad 0.116$

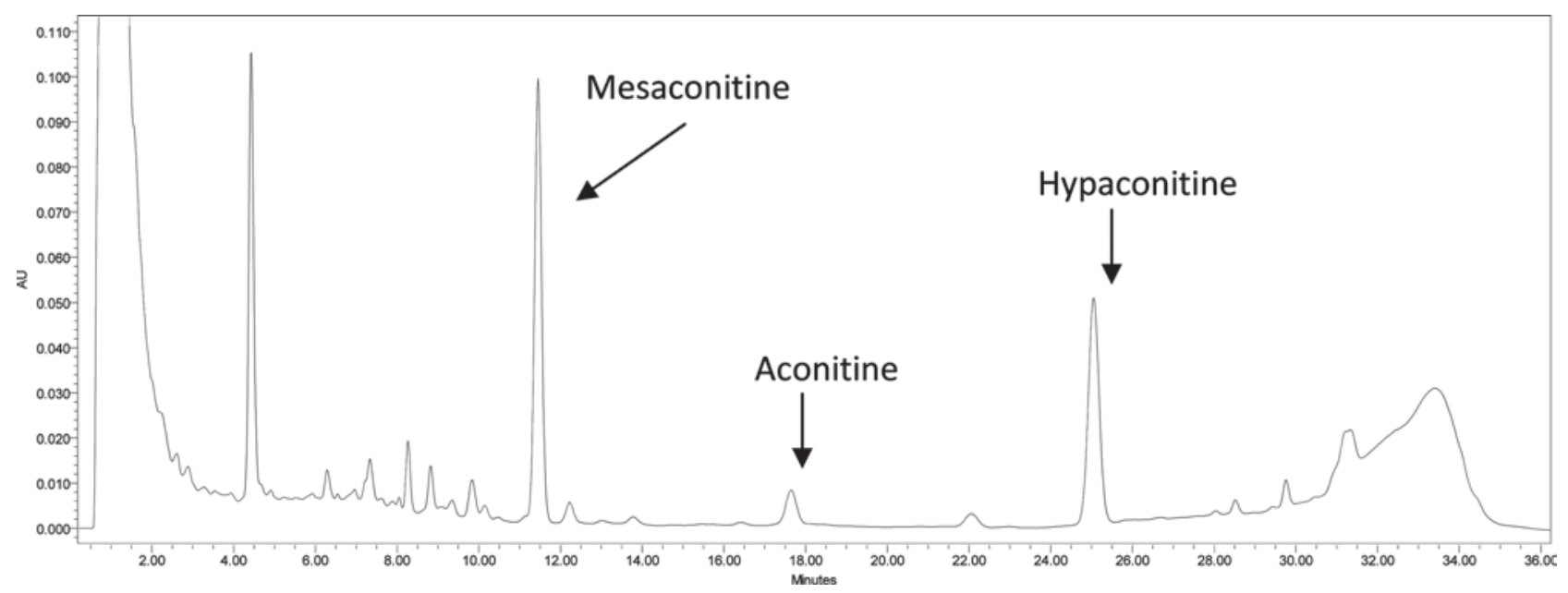

Figure 3. HPLC chromatogram of the extract prepared from a crude aconite root sample. 


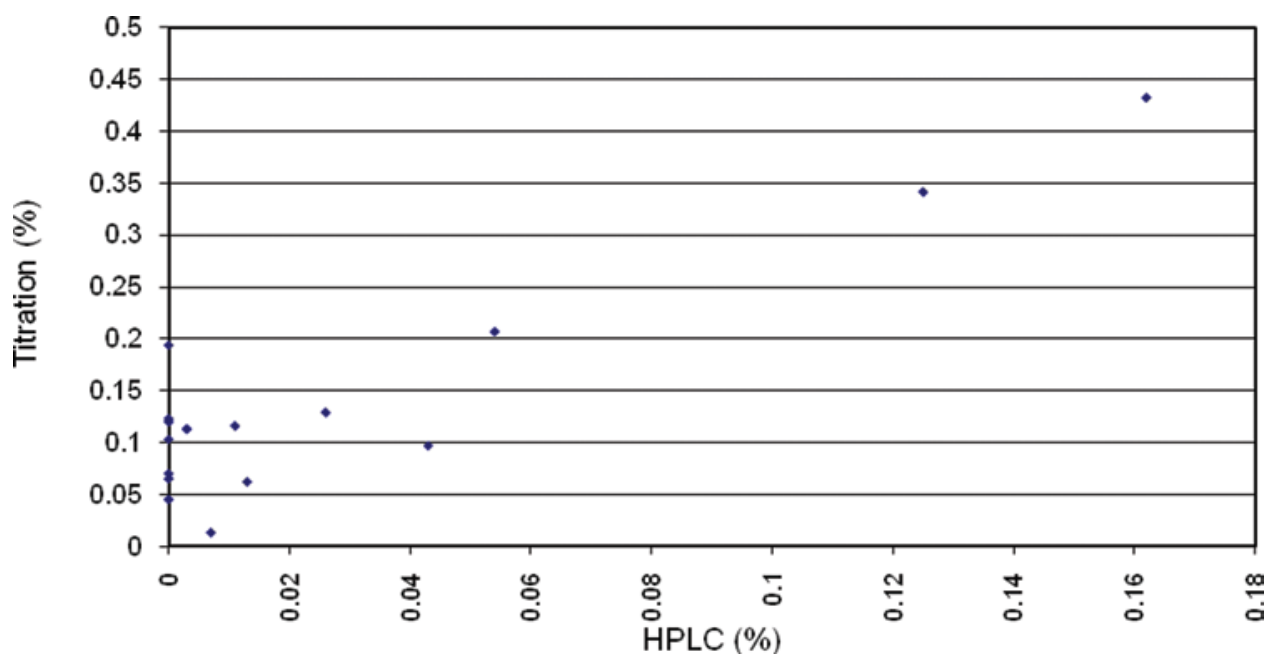

Figure 4. Correlation of the alkaloid contents by HPLC quantification and titration.

far; therefore, it is not possible to standardize these plant materials to any active agent.

\section{Conclusions}

Alkaloid titration, a method applied in the quality control of homeopathic aconite tinctures and aconite drugs of the Chinese Pharmacopoeia, may give information on total content of alkaloids, but allows no conclusion on the content of toxic alkaloids. Therefore, quantification of diester-type diterpene alkaloids is essential to ensure the safety of medicines based on these herbal drugs.

The sample preparation and HPLC analysis method developed by us offers a quick and reliable possibility to determine the quantity of the most important toxic alkaloids of A. carmichaeli and A. kusnezoffii and should now be considered as a purity test in pharmacopoeias. The relevance of our method was confirmed in in vivo toxicity studies, which exhibited an apparent correlation between the alkaloid content and the severity of toxic symptoms.

\section{Acknowledgement}

This project was supported by Future Funds Styria (PN 4071), Austrian Federal Ministry of Science and Research, Austrian Federal Ministry of Health, and Eurasia Pacific UniNet.

\section{Declaration of interest}

The authors report no declarations of interest.

\section{References}

Ameri A. (1998). The effects of Aconitum alkaloids on the central nervous system. Prog Neurobiol, 56, 211-235.

Caravati EM, McCowan CL, Marshall SW. (2004). Plants. In: Dart RC, ed. Medical Toxicology. Philadelphia: Lippincott Williams \& Wilkins, 1701.
Chan TY. (2009). Aconite poisoning. Clin Toxicol (Phila), 47, 279-285. Chinese Pharmacopoeia Commission. (2005). Pharmacopoeia of the People's Republic of China. Beijing: People's Medical Publishing House.

Csupor D, Wenzig EM, Zupkó I, Wölkart K, Hohmann J, Bauer R. (2009). Qualitative and quantitative analysis of aconitine-type and lipo-alkaloids of Aconitum carmichaelii roots. J Chromatogr A, 1216, 2079-2086.

EDQM homepage: http://www.edqm.eu/en/Knowledge-Database707.html. Accessed on 22 December 2010.

German Homeopathic Pharmacopoeia, Volume 1. (2005). Medpharm Scientific Publishers Stuttgart.

-Gutser UT, Friese J, Heubach JF, Matthiesen T, Selve N, Wilffert B, Gleitz J. (1998). Mode of antinociceptive and toxic action of alkaloids of Aconitum spec. Naunyn Schmiedebergs Arch Pharmacol, 357, 39-48.

-Jiang ZH, Xie Y, Zhou H, Wang JR, Liu ZQ, Wong YF, Cai X, Xu HX, Liu L. (2005). Quantification of Aconitum alkaloids in aconite roots by a modified RP-HPLC method. Phytochem Anal, 16, 415-421.

KangXQ, FanZC,ZhangZQ.(2010). Simultaneous determination of three Aconitum alkaloids in six herbal medicines by high-performance liquid chromatography. J Chromatogr Sci, 48, 860-865.

Pharmacopoeia Helvetica, Ed. VII. (1988). Bern: Office Central Fdral des Imprims et du Matriel, p. 1463.

Singhuber J, Zhu M, Prinz S, Kopp B. (2009). Aconitum in traditional Chinese medicine: A valuable drug or an unpredictable risk? J Ethnopharmacol, 126, 18-30.

Wada K, Nihira M, Ohno Y. (2006). Effects of chronic administrations of aconitine on body weight and rectal temperature in mice. J Ethnopharmacol, 105, 89-94.

Wang Y, Liu Z, Song F, Liu S. (2002). Electrospray ionization tandem mass spectrometric study of the aconitines in the roots of aconite. Rapid Commun Mass Spectrom, 16, 2075-2082.

Wang Y, Shi L, Song F, Liu Z, Liu S. (2003). Exploring the esterexchange reactions of diester-diterpenoid alkaloids in the aconite decoction process by electrospray ionization tandem mass spectrometry. Rapid Commun Mass Spectrom, 17, 279-284.

-Wang Z, Wen J, Xing J, He Y. (2006). Quantitative determination of diterpenoid alkaloids in four species of Aconitum by HPLC. J Pharm Biomed Anal, 40, 1031-1034.

Xiao PG, Wang FP, Gao F, Yan LP, Chen DL, Liu Y. (2006). A pharmacophylogenetic study Aconitum L. (Ranunculaceae) from China. Acta Phytotaxon Sin, 44, 1-46.

Xie Y, Jiang ZH, Zhou H, Xu HX, Liu L. (2005). Simultaneous determination of six Aconitum alkaloids in proprietary Chinese medicines by high-performance liquid chromatography. J Chromatogr A, 1093, 195-203. 